\title{
Capsule Commentary on Patel el al., Using Active Choice Within the Electronic Health Record to Increase Influenza Vaccination Rates
}

\author{
Jason M. Beckta, MD, PhD \\ Yale School of Medicine, New Haven, CT, USA. \\ J Gen Intern Med 32(7):800 \\ DOI: $10.1007 / \mathrm{s} 11606-017-4057-3$ \\ () Society of General Internal Medicine 2017
}

P atel et al. ${ }^{1}$ examine the effect of an "active choice" prompt in an electronic health record (EHR) system on influenza vaccination rates during outpatient clinic appointments. For two influenza seasons (2010-2011 and 20112012), the vaccine administration rate was recorded for three similar clinics. During the 2012-2013 season, two of the three clinics were held as controls, while the EHR for the third clinic was modified to require both the physician and assistant to choose between accepting or canceling an automatic vaccine order. The authors report a significant increase in the rates of vaccination for the active choice group. ${ }^{1}$ Based on these results, the authors conclude that an active choice prompt has the potential to increase the rates of vaccination as well as the utilization of other preventative interventions.

Though producing convincing data, the study was unavoidably complicated by the natural variation in the severity of influenza seasons. Per the Centers for Disease Control and Prevention (CDC), the 2012-2013 season was "moderately severe" compared to previous years. ${ }^{2}$ Additionally, the peak national influenza activity in the experimental year was late December, with $38.2 \%$ of patients testing positive. ${ }^{2}$ This was in stark contrast to the previous year's peak in mid-March, with only $2.4 \%$ of patients testing positive. These seasonal variations are reflected in the study's data, as both the intervention and control groups had a significant increase in immunization rates compared to the pre-intervention time period.
The authors discuss other studies which demonstrate that too many EHR prompts can result in "alert fatigue," and the beneficial effect of these reminders can be lost. ${ }^{3}$ Unfortunately, the experimental year occurred during a severe influenza season with an early peak, effectively creating an environment of heightened awareness, within which both patients and providers are more cognizant of vaccination status. The relative vaccination rate increase in the active choice clinic, while statistically greater, was small $(6.6 \%, 95 \%$ CI: $5.5-8.1)$. Whether the difference would have been greater in the absence of an early and severe flu season is uncertain. Additional research spanning several seasons is needed to better understand the effectiveness of the active choice prompt.

Corresponding Author: Jason M. Beckta, MD, PhD; Yale School of Medicine, New Haven, CT, USA (e-mail: jason.beckta@yale.edu).

Compliance with Ethical Standards:

Conflict of Interest: The author has no conflicts of interest with this article.

\section{REFERENCES}

1. Patel MS, Volpp KG, Small DS, et al. Using Active Choice within the Electronic Health Record to Increase Influenza Vaccination Rates. J Gen Intern Med. (2017). doi: 10.1007/s11606-017-4046-6

2. The 2012-2013 Influenza Season. Centers for Disease Control and Prevention. https://www.cdc.gov/flu/pastseasons/1213season.htm. Published March 10, 2015. Accessed 30 Mar 2017.

3. Black AD, Car J, Pagliari C, et al. The impact of eHealth on the quality and safety of health care: a systematic overview. PLoS Med. 2011;8:e1000387. 